\title{
OPERATIONAL CALCULUS FOR THE CONTINUOUS LEGENDRE TRANSFORM WITH APPLICATIONS
}

\author{
E.Y. DEEBA \\ Department of Applied Mathematical Sciences \\ University of Houston-Downtown \\ Houston, Texas 77002 \\ E.L. KOH \\ Department of Mathematics and Statistics \\ University of Regina \\ Regina, Saskatchewan, Canada, S4S 0A2
}

(Received June 13, 1988 and in revised form September , 1988)

ABSTRACT. This paper develops an operational calculus for the continuous Legendre transform introduced and studied by Butzer, Stens and Wehrens [1]. It is an extension of the work done by Churchill et al [2], [3] for the discrete case. In particular, a differentiation theorem and a convolution theorem are proved and the results are applied to the solution of some boundary value problems.

KEY WORDS AND PHRASES. Continuous Legendre Transform, Operational Calculus, Convolution, Boundary Value Problems.

1980 AMS SUBJECT CLASSIFICATION CODES: 4 4A15, 99A90, 44A20.

1. INTRODUCTION. For a given function $f$ belonging to an appropriate function space, the continuous Legendre transform is defined by

$$
(T f)(\lambda)=\frac{1}{2} \int_{-1}^{1} P_{\lambda}(x) f(x) d x
$$

where $P_{\lambda}(x)$ is the Legendre function and $\lambda \geq-\frac{1}{2}$. This transform has been introduced and studied by Butzer, Stens and Wehrens [1]. The discrete analog of the transform in (1) has been studied by Churchill [2] and Churchill and Dolph [3]. The object of this paper is to develop an operational calculus for the transform which is useful in solving partial differential equations whose underlying differential form is given by

$$
D=\frac{d}{d x}\left[\left(1-x^{2}\right) \frac{d}{d x}\right] .
$$

In section 2 we present the background material needed in the sequel. In section 3 , we derive the operational calculus for (1) including a convolution theorem and a table of transforms of some functions. In the last section we apply the results to solving some boundary value problems. 
2. PRELIMINARIES. We recall basic properties of the transform $(T f)(\lambda)$ (see [1]) and important contiguous relations that hold for the Legendre function.

The Legendre function $P_{\lambda}(x)$ is given by

$$
P_{\lambda}(x)={ }_{2} F_{1}\left(-\lambda, \lambda+1 ; 1 ; \frac{1-x}{2}\right)=\sum_{k=0}^{\infty} \frac{(-\lambda)_{k}(\lambda+1)_{k}}{(k !)^{2}}\left(\frac{1-x}{2}\right)^{k}, x \varepsilon(-1,1] .
$$

Since $P_{\lambda-1}(x)=P_{-\lambda}(x)$, it sufficies to consider the case $\lambda \geq-\frac{1}{2} . P_{\lambda}(x)$ satisfies the differential equation

$$
D y+\lambda(\lambda+1) y=0
$$

where $D$ is as given in (2). Further, it satisfies the relations $P_{\lambda}(1)=1, P_{\lambda}^{\prime}(1)=\frac{\lambda(\lambda+1)}{2}, \lim _{x \rightarrow-1^{+}}(1+$ $x) P_{\lambda}(x)=0$ and $\lim _{x \rightarrow-1^{+}}(1+x) P_{\lambda}^{\prime}(x)=\frac{\sin \pi \lambda}{\pi}$.

The following contiguous relations (see [4]) will be useful in the derivation of the calculus for $(T f)(\lambda)$ :

$$
(2 \lambda+1) x P_{\lambda}(x)=(\lambda+1) P_{\lambda+1}(x)+\lambda P_{\lambda-1}(x)
$$

and

$$
\left(1-x^{2}\right) P_{\lambda}^{\prime}(x)=-\lambda x P_{\lambda}(x)+\lambda P_{\lambda-1}(x) .
$$

From (3) and (4) we obtain the relation

$$
\left(1-x^{2}\right) P_{\lambda}^{\prime}(x)=-\frac{\lambda(\lambda+1)}{2 \lambda+1}\left(P_{\lambda+1}(x)-P_{\lambda-1}(x)\right) .
$$

The addition formula for the Legendre functions (see [4]) is given by

$$
P_{\lambda}(\cos \alpha) P_{\lambda}(\cos \beta)=P_{\lambda}(\cos \nu)-2 \sum_{m=1}^{\infty} \frac{\Gamma(\lambda-m+1)}{\Gamma(\lambda+m+1)} P_{\lambda}^{m}(\cos \alpha) P_{\lambda}^{m}(\cos \beta) \cos m \gamma
$$

where $P_{\lambda}^{m}(\cdot)$ is the associated Legendre function and $\cos \nu=\cos \alpha \cos \beta+\sin \alpha \sin \beta \cos \gamma$ with $0 \leq \alpha, \beta \leq \pi, \alpha+\beta<\pi, \gamma$ real. Formula (6) will be useful in deriving the convolution theorem. Another useful relation involving the Legendre functions is

$$
\int_{-1}^{1} P_{\lambda}(x) P_{\nu}(-x) d x=\frac{\sin \pi \lambda-\sin \pi \nu}{\pi(\lambda-\nu)(\lambda+\nu+1)}, \lambda \neq \nu, \lambda+\nu+1 \neq 0 .
$$

The Legendre transform $(T f)(\lambda)$ is a linear integral transform from $L_{2}(-1,1]$ into the space $C_{0}(-1,1] \cap L_{2}(-1,1]$. For $f \varepsilon L_{2}(-1,1]$, it was shown in [1] that $(T f)(\lambda)=0\left(\lambda^{-\frac{1}{2}}\right)$ as $\lambda \rightarrow \infty$ and $(T f)\left(\lambda-\frac{1}{2}\right) \varepsilon C_{0}(-1,1] \cap L_{2}(-1,1]$. Further, it was shown that if $f \varepsilon L_{2}(-1,1] \cap C(-1,1]$ and if $\sqrt{\lambda}(T f)\left(\lambda-\frac{1}{2}\right) \varepsilon L_{1}\left(\mathbf{R}^{+}\right)$, then the inversion formula is given by

$$
f(x)=T^{-1}((T f)(\lambda))=4 \int_{0}^{\infty}(T f)\left(\lambda-\frac{1}{2}\right) P_{\lambda-\frac{1}{2}}(-x) \lambda \sin \pi \lambda d \lambda
$$

\section{BASIC OPERATIONAL PROPERTIES FOR $(T f)(\lambda)$. In this section we slall}

operational calculus for the continuous Legendre transform $(T f)(\lambda)$ thus exterding the firulw. obtained by Churchill [2] and Churchill and Dolph [3] for the discrete case. Wr shall alw deriw the Legendre transform of some functions.

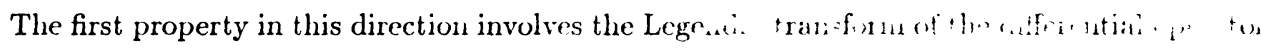
$D$ as given in (2). 
Theorem 3.1. Let $f$ be a function such that (i) $f^{(k)} \varepsilon C(-1,1] \cap L_{2}(-1,1] \quad k=0,1$ (ii) $\lim _{x \rightarrow \pm 1}\left(1-x^{2}\right) f(x)=\lim _{x \rightarrow \pm 1}\left(1-x^{2}\right) f^{\prime}(x)=0$ and (iii) $(T f)(\lambda)$ exists. Then

$$
(T(D f))(\lambda)=-\lambda(\lambda+1)(T f)(\lambda) .
$$

Proof. From (1) together with successive integration by parts, we obtain

$$
\begin{aligned}
(T(D f))(\lambda) & =\frac{1}{2} \int_{-1}^{1} P_{\lambda}(x) D f(x) d x \\
& =\frac{1}{2} \int_{-1}^{1} P_{\lambda}(x) \frac{d}{d x}\left[\left(1-x^{2}\right) \frac{d}{d x} f(x)\right] d x \\
& =\left[\frac{1}{2} P_{\lambda}(x)\left(1-x^{2}\right) f^{\prime}(x)-\frac{1}{2} P_{\lambda}^{\prime}(x)\left(1-x^{2}\right) f(x)\right]_{-1^{+}}^{+1^{-}} \\
& -\frac{1}{2} \lambda(\lambda+1) \int_{-1}^{1} P_{\lambda}(x) f(x) d x .
\end{aligned}
$$

The result follows from the facts that $P_{\lambda}(1)=1, P_{\lambda}^{\prime}(1)=\frac{\lambda(\lambda+1)}{2}, \lim _{x \rightarrow-1^{+}}(1+x) P_{\lambda}(x)=0$ and $\lim _{x \rightarrow-1^{+}}(1+x) P_{\lambda}^{\prime}(x)=\frac{\sin \pi \lambda}{\pi}$ together with the hypothesis (ii).

This basic operational property reduces a given differential equation which involves the operator $D$ into an algebraic one or into a differential equation with one less independent variable.

Remark 3.1. (a) If, in Theorem $3.1, D^{k} f=D^{k-1}(D f)$ and $f^{(k)}$ satisfy the same hypotheses, then

$$
T\left(\left(D^{k} f(x)\right)\right)(\lambda)=(-1)^{k} \lambda^{k}(\lambda+1)^{\lambda}(T f)(\lambda), k=1,2, \ldots
$$

(b) We note that (9) can be cast into the form

$$
\frac{1}{4}(T f)(\lambda)-T((D f))(\lambda)=\left(\lambda+\frac{1}{2}\right)^{2}(T f)(\lambda)
$$

The second operational property involves the relationship between the transform of a given function $f$ and the function $g(x)=\int_{-1}^{x} f(t) d t$.

Theorem 3.2. If $f$ is a piecewise continuous function defined on $(-1,1)$ and $g(x)=\int_{-1}^{x} f(t) d t$ and if $(T f)(\lambda)$ exists, then

$$
(T g)(\lambda)=-\frac{(T f)(\lambda+1)-(T f)(\lambda-1)}{2 \lambda+1}
$$

Proof. Since $D\left(P_{\lambda}(x)\right)=-\lambda(\lambda+1) P_{\lambda}(x)$, it follows that

$$
\begin{aligned}
(T g)(\lambda) & =-\frac{1}{2 \lambda(\lambda+1)} \int_{-1}^{1} \frac{d}{d x}\left[\left(1-x^{2}\right) \frac{d}{d x} P_{\lambda}(x)\right] g(x) d x \\
& =-\left.\frac{1}{2 \lambda(\lambda+1)}\left(1-x^{2}\right) P_{\lambda}^{\prime}(x) g(x)\right|_{-1} ^{1}+\frac{1}{2 \lambda(\lambda+1)} \int_{-1}^{1}\left(1-x^{2}\right) P_{\lambda}^{\prime}(x) f(x) d x .
\end{aligned}
$$

Since $P_{\lambda}^{\prime}(1)$ and $g(1)$ are defined, $g(-1)=0$ and $\lim _{x \rightarrow-1^{+}}(1+x) P_{\lambda}^{\prime}(x)=\frac{\sin \pi \lambda}{\pi}$, the first 1 in :identically zero. Thus

$$
(T g)(\lambda)=\frac{1}{2 \lambda(\lambda+1)} \int_{-1}^{1}\left(1-x^{2}\right) P_{\lambda}^{\prime}(x) f(x) d x .
$$

The contiguous relation (5) will then imply that

$$
(T g)(\lambda)=\frac{1}{2 \lambda(\lambda+1)} \int_{-1}^{1}-\frac{\lambda(\lambda+1)}{2 \lambda+1}\left(P_{1, i},-P_{i},(1)\right) f(r) \cdot h
$$


Equivalently,

$$
(T g)(\lambda)=-\frac{(T f)(\lambda+1)-(T f)(\lambda-1)}{2 \lambda+1} .
$$

Remark 3.2. Similar difference relations to that of (11) can be obtained in the following situation.

(a) If $g(x)=x f(x)$ and if $(T f)(\lambda)$ exists, then under appropriate conditions on $f$, one obtains

$$
(T g)(\lambda)=\frac{(\lambda+1)(T f)(\lambda+1)+\lambda(T f)(\lambda-1)}{2 \lambda+1}
$$

This will follow by applying the contiguous relation (3).

(b) If $g(x)=\int_{-1}^{x}(x-t) f(t) d t$ and if $(T f)(\lambda)$ exists, then, again under appropriate conditions on $f$, the contiguous relation (5) and Theorem 3.2 yields

$$
(T g)(\lambda)=\frac{(T f)(\lambda+2)-2(T f)(\lambda)+(T f)(\lambda-2)}{(2 \lambda+1)^{2}}
$$

The next operational property that we will derive involves the inverse of the differential operator $D$. We define the inverse of $D$, denoted by $D^{-1}$, by $D^{-1}(f(x))=g(x)$ if and only if $D(g(x))=f(x)$. If $(T f)(\lambda)$ is known, then we want to relate $T\left(\left(D^{-1} f\right)\right)(\lambda)$ to the transform of $f$.

If, for a given function $f(x), D(g(x))=f(x)$, then on integrating twice, we obtain

$$
g(x)=\int_{0}^{x} \frac{1}{1-t^{2}} \int_{-1}^{t} f(\alpha) d \alpha d t+c
$$

for some constant c. If $f(x)$ is in addition an even function on $(-1,1)$, then one can show by employing a continuity argument that $\lim _{x \rightarrow \pm 1}\left(1-x^{2}\right) g(x)=\lim _{x \rightarrow \pm 1}\left(1-x^{2}\right) g^{\prime}(x)=0$. Theorem 3.1 will then imply that

$$
(T f)(\lambda)=T((D g))(\lambda)=-\lambda(\lambda+1)(T g)(\lambda) .
$$

Equivalently,

$$
(T g)(\lambda)=-\frac{1}{\lambda(\lambda+1)} T((D g))(\lambda)=-\frac{(T f)(\lambda)}{\lambda(\lambda+1)}
$$

Thus

$$
T\left(D^{-1} f\right)(\lambda)=-\frac{1}{\lambda(\lambda+1)}(T f)(\lambda) .
$$

This last relation implies that $D^{-1} f$ is the inverse Legendre transform of $-\frac{(T f)(\lambda)}{\lambda(\lambda+1)}$. We thus have

Theorem 3.3. If $f(x)$ is such that $f(x)$ is even on $(-1,1), f \varepsilon L_{2}(-1,1] \cap C(-1,1],(T f)(\lambda)$ exists and $\frac{(T f)(\lambda)}{\sqrt{\lambda(\lambda+1)}} \varepsilon L_{1}\left(\mathbf{R}^{+}\right)$, then

$$
D^{-1}(f(x))=T^{-1}\left(-\frac{(T f)(\lambda)}{\lambda(\lambda+1)}\right)
$$

where the inverse transform $T^{-1}$ is given by (8).

We shall finally develop a convolution property for the Legendre transform. In particular, we will show 
Theorem 3.4. If $f(x)$ and $g(x)$ are given functions for which $(T f)(\lambda)$ and $(T g)(\lambda)$ respectively exist, then their product $(T f)(\lambda)(T g)(\lambda)$ is the transform of the function $h(x)=f(x) * g(x)$ where $h(x)$ is given by

$$
h(\cos \nu)=\frac{1}{2 \pi} \int_{0}^{\pi} \int_{0}^{\pi} f(\cos \alpha) g(\cos \beta) \sin \alpha d \alpha d \theta
$$

where $\cos \beta=\cos \alpha \cos \nu+\sin \alpha \sin \nu \cos \theta$ with $0 \leq \alpha, \nu \leq \pi, \alpha+\nu<\pi$ and $\theta$ is real. The variables $\alpha, \nu$ and $\beta$ may be interpreted as the sides of a splierical triangle on the unit hemisphere and $\theta$ is the angle between the sides $\alpha$ and $\nu$ (see Figure 1).

Proof. From (1), we have

$$
(T f)(\lambda)(T g)(\lambda)=\frac{1}{4} \int_{-1}^{1} P_{\lambda}(x) f(x) d x \int_{-1}^{1} P_{\lambda}(y) g(y) d y .
$$

Set $x=\cos \alpha$ and $y=\cos \beta$. Then

$$
(T f)(\lambda)(T g)(\lambda)=\frac{1}{4} \int_{0}^{\pi} f(\cos \alpha) \sin \alpha \int_{0}^{\pi} P_{\lambda}(\cos \alpha) P_{\lambda}(\cos \beta) g(\cos \beta) \sin \beta d \beta d \alpha .
$$

The addition formula for the Legendre function (G) will yield upon an integration with respect to $\gamma$ from 0 to $\pi$

$$
P_{\lambda}(\cos \alpha) P_{\lambda}(\cos \beta)=\frac{1}{\pi} \int_{0}^{\pi} P_{\lambda}(\cos \nu) d \gamma
$$

where $\cos \nu=\cos \alpha \cos \beta+\sin \alpha \sin \beta \cos \gamma$ (see figure 1).

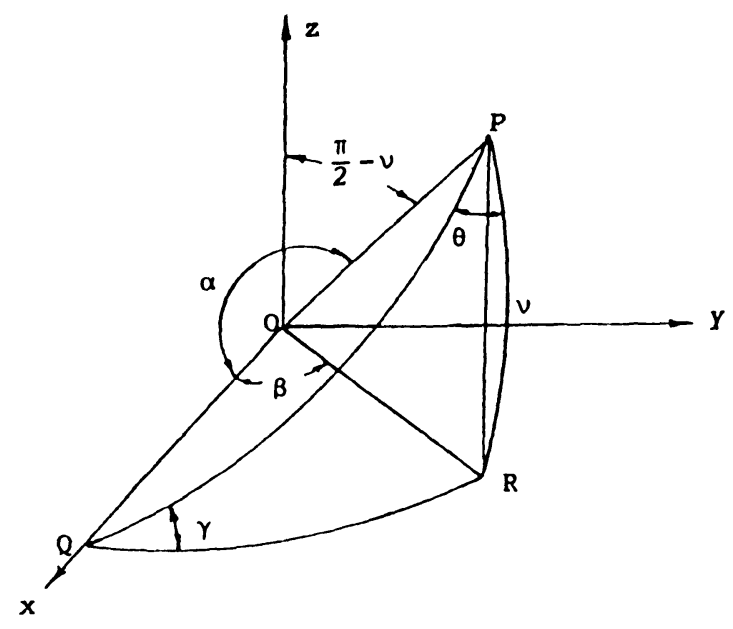

Figure 1

Thus

$$
(T f)(\lambda)(T g)(\lambda)=\frac{1}{4 \pi} \int_{0}^{\pi} f(\cos \alpha) \sin \alpha \int_{0}^{\pi} \int_{0}^{\pi} P_{\lambda}(\cos \nu) g(\cos \beta) \sin \beta d \gamma d \beta d \alpha .
$$

In the spherical triangle $P Q R$, we have

$$
\cos \beta=\cos \alpha \cos \nu+\sin \alpha \sin \nu \cos \theta .
$$

Using this relation along with the sine law and transformation of co-ordinates, the double integral can be written as: 


$$
\int_{0}^{\pi} \int_{0}^{\pi} P_{\lambda}(\cos \nu) g(\cos \beta) \sin \nu d \theta d \nu
$$

Hence,

$$
(T f)(\lambda)(T g)(\lambda)=\frac{1}{2} \int_{0}^{\pi} P_{\lambda}(\cos \nu) \sin \nu\left[\frac{1}{2 \pi} \int_{0}^{\pi} \int_{0}^{\pi} f(\cos \alpha) g(\cos \beta) \sin \alpha d \alpha d \theta\right] d \nu .
$$

The expression in the bracket is a function of $\nu$ and we then write

$$
h(\cos \nu)=\frac{1}{2 \pi} \int_{0}^{\pi} \int_{0}^{\pi} f(\cos \alpha) g(\cos \beta) \sin \alpha d \alpha d \theta
$$

This may be interpreted as a convolution product of $f$ and $g$ and $(T h(\cos \nu))(\lambda)=(T f)(\lambda)(T g)(\lambda)$. This proves Theorem 3.4.

Geometrically, the expression (15) is the mean value of $f(\cos \alpha) g(\cos \beta)$ over the unit hemisphere $x^{2}+y^{2}+z^{2}=1, z \geq 0$. To see this, we note that the element surface area is $d S=\sin \alpha d \alpha d \theta$. This is clear if we identify the coordinate transformation in Figure 1 by

$$
\begin{aligned}
& x=\cos \alpha \\
& y=\sin \alpha \sin \theta \\
& z=\sin \alpha \cos \theta
\end{aligned}
$$

Thus (15) reads

$$
h(\cos \nu)=\frac{1}{2 \pi} \int_{S} \int f(\cos \alpha) g(\cos \beta) d S .
$$

We will now evaluate the Legendre transform of some functions.

1. $f(x)=$ constant $=k$

$$
(T f)(\lambda)= \begin{cases}k \frac{\sin \pi \lambda}{\pi \lambda(\lambda+1)} & \lambda \neq 0 \\ k & \lambda=0\end{cases}
$$

2. $f(x)=P_{n}(x)$. Then by (2.5) we have, for $n=0,1,2, \ldots$,

$$
\begin{aligned}
(T f)(\lambda) & =\frac{1}{2} \int_{-1}^{1} P_{\lambda}(x) P_{n}(x)=\frac{(-1)^{n}}{2} \int_{-1}^{1} P_{\lambda}(x) P_{n}(-x) d x \\
& =\frac{\sin \pi(\lambda-n)}{2 \pi(\lambda-n)(\lambda+n+1)}, \lambda \neq n,-(n+1) .
\end{aligned}
$$

3. $f(x)=\log (1-x)$.

$$
\begin{aligned}
(T f)(\lambda) & =\frac{1}{2} \int_{-1}^{1} P_{\lambda}(x) \log (1-x) d x \\
& =-\frac{1}{2 \lambda(\lambda+1)} \int_{-1}^{1} \frac{d}{d x}\left[\left(l-x^{2}\right) \frac{d}{d x} P_{\lambda}(x)\right] \log (1-x) d x \\
& =(\log 2) \frac{\sin \pi \lambda}{\lambda(\lambda+1)}-\frac{1}{\lambda(\lambda+1)}-\frac{1}{2 \lambda(\lambda+1)} \int_{-1}^{1} P_{\lambda}(x) \frac{d}{d x}\left[\left(1-x^{2}\right) \frac{d}{d x} \log (1-x)\right] d x .
\end{aligned}
$$

Observe that $D(\log (1-x))=\frac{d}{d x}\left[\left(1-x^{2}\right) \frac{d}{d x} \log (1-x)\right]=-1$. Thus

$$
(T f)(\lambda)=(\log 2) \frac{\sin \pi \lambda}{\lambda(\lambda+1)}-\frac{1}{\lambda(\lambda+1)}-\frac{\sin \pi \lambda}{\lambda^{2}(\lambda+1)^{2}}
$$

4. $f(\lambda)=\int_{-1}^{x} \frac{1}{1-t} d t$. By using 1 and 3 above, we obtain 


$$
(T f)(\lambda)=\frac{1}{\lambda(\lambda+1)}+\frac{\sin \pi \lambda}{\lambda^{2}(\lambda+1)^{2}} .
$$

5. $f(x)=\left(1-2 t x+x^{2}\right)^{-\frac{1}{2}}=\sum_{n=0}^{\infty} t^{n} P_{n}(x),-1<t<1$. From (2) above

$$
(T f)(\lambda)=\frac{\sin \pi \lambda}{2 \pi} \sum_{n=0}^{\infty} \frac{t^{n}}{(\lambda-n)(\lambda+n+1)} .
$$

We finally remark that for $\lambda$ equal to a non- negative integer, the results of this section yield those obtained in [2] and [3].

4. APPLICATIONS. In this section we consider some applications of the Legendre transform. We consider problems arising in heat conduction and in potential theory.

A. Heat Conduction Problem. Consider a non-homogeneous bar with extremities at $x= \pm 1$ and is insulated at these end points. Let $u(x, t)$ be the temperature of the bar at position $x$ at time $t$. The one dimensional heat equation with prescribed initial temperature is given by

$$
\begin{aligned}
\frac{\partial}{\partial x}\left(k \frac{\partial}{\partial x} u(x, t)\right) & =\rho c \frac{\partial u}{\partial t}(x, t) & \\
u(x, 0) & =g(x), & -1<x<1
\end{aligned}
$$

where $k, \rho$ and $c$ are physical constants representing thermal conductivity, density and specific heat respectively. We assume that the thermal conductivity $k$ is given by $k=\alpha\left(1-x^{2}\right), \alpha$ being a real constant. The above equation reads

$$
\begin{aligned}
\frac{\partial}{\partial x}\left(\left(1-x^{2}\right) \frac{\partial}{\partial x} u(x, t)\right) & =\frac{\rho c}{\alpha} \frac{\partial u}{\partial t}(x, t) & \\
u(x, 0) & =g(x), & -1<x<1 .
\end{aligned}
$$

If $U(\lambda, t)=T(u(x, t))(\lambda)$ and $G(\lambda)=(T u(x, 0))(\lambda)$, then, by Theorem 3.1, we obtain upon the application of the transform

$$
\begin{aligned}
\frac{d}{d t} U(\lambda, t) & =-\frac{\alpha}{\rho c} \lambda(\lambda+1) U(\lambda, t) \\
U(\lambda, 0) & =G(\lambda) .
\end{aligned}
$$

The solution is given by

$$
U(\lambda, t)=G(\lambda) e^{-\frac{a}{\rho c}(\lambda+1) \lambda t}
$$

Now $u(x, t)$ can be obtained by either employing the inversion formula (8) or the convolution theorem. By employing the inversion formula and under the assumption that $u(x, t) \varepsilon C(-1,1] \cap$ $L_{2}(-1,1)$ and $\sqrt{\lambda} U\left(\lambda-\frac{1}{2}, t\right) \varepsilon L_{1}\left(R^{+}\right)$, one obtains

$$
u(x, t)=4 \int_{0}^{\infty} G\left(\lambda-\frac{1}{2}\right) e^{-\frac{a}{\rho c}\left(\lambda^{2}-\frac{1}{4}\right) t} P_{\lambda}(-x) \lambda \sin \pi \lambda d \lambda
$$

On the other hand the convolution property (Theorem 3.4) will yield

$$
u(\cos \nu, t)=\frac{1}{2 \pi} \int_{0}^{\pi} \int_{0}^{\pi} g(\cos \alpha) f(\cos \beta) \sin \alpha d \alpha d \theta
$$

where $\alpha, \beta, \theta$ are as in Figure 1 and $\cos \beta=\cos \alpha \cos \nu+\sin \alpha \sin \nu \cos \theta$ and $f$ is the inverse transform of $e^{-\frac{\alpha}{\rho c}\left(\lambda^{2}-\frac{1}{4}\right) t}$. That is, by (8)

$$
f(x)=e^{\frac{a}{4 \rho c} t} 4 \int_{0}^{\infty} e^{-\frac{a}{\rho c}\left(\lambda-\frac{1}{2}\right)^{2}} P_{\lambda}(-x) \lambda \sin \pi \lambda d \lambda .
$$


B. Dirichlet Problem for the Unit Sphere (see[2]) Consider the problem of determining the potential $v(r, \cos \theta)$ in the interior of a unit sphere with a prescribed potential $f(\cos \theta)$ on $r=$ $1,0 \leq \theta \leq \pi$. The Laplace equation defining this potential is

$$
\nabla^{2} v=\frac{1}{r}(r v)_{r r}+\frac{1}{r^{2} \sin \theta}\left(\sin \theta v_{\theta}\right)_{\theta}=0
$$

If $x=\cos \theta$, then the equation reduces to

$$
\begin{aligned}
r(r v)_{r r}+\left(\left(1-x^{2}\right) v_{x}\right)_{x} & =0 \\
v(1, x) & =f(x), \quad-1 \leq x \leq 1 .
\end{aligned}
$$

If $V(r, \lambda)$ and $F(\lambda)$ denote respectively the Legendre transform of $v(r, x)$ and $f(x)$, then, upon applying the transform to the underlying equation, we obtain

$$
\begin{aligned}
\left.r \frac{d^{2}}{d r^{2}}(r V(r, \lambda))-\lambda(\lambda+1)\right) V(r, \lambda) & =0, \\
V(1, \lambda) & =F(\lambda) .
\end{aligned}
$$

The solution of this equation is given by

$$
V(r, \lambda)=c_{1} r^{\lambda}+c_{2} r^{-(\lambda+1)}
$$

In order to apply the inversion formula (8) we need to have $v(r, x) \varepsilon L_{2}(-1,1] \cap C(-1,1]$ and $\sqrt{\lambda} V(r, \lambda) \varepsilon L_{1}\left(\mathbf{R}^{+}\right)$. This will imply that $c_{2}=0$ and $v(1, \lambda)=F(\lambda)$ will imply that $c_{1}=F(\lambda)$. Hence the solution is given by

$$
v(r, \lambda)=F(\lambda) r^{\lambda}
$$

and

$$
v(r, x)=r \int_{0}^{\infty} F\left(\lambda-\frac{1}{2}\right) r^{\lambda-\frac{1}{2}} P_{\lambda}(-x) \lambda \sin \pi \lambda d \lambda .
$$

ACKNOWLEDGEMENT. The work of the first author was partially supported by a research grant from the University of Houston-Downtown. The second author was partially supported by NSERC of Canada under Grant A-7184.

\section{REFERENCES}

1. Butzer, P.L., R.L. Stens and M. Wehrens, The continuous Legendre transform, its inverse transform and applications, Internat. J. Math and Math. Sciences, Vol. 3, No. 1 (1980), 47-67.

2. Churchill, R.V., The operational calculus of the Legendre transform, J. Math. \& Phys., 33$(1954), 165-177$.

3. Churchill, R.V. and C.L. Dolph, Inverse transforms of product of Legendre transforms, Proc. Amer. Math. Soc., 5

4. Erdelyi, A., et al, Higher Transcendental Functions, Vol. 1, Mclisaw Hill, Z..'., 1953. 


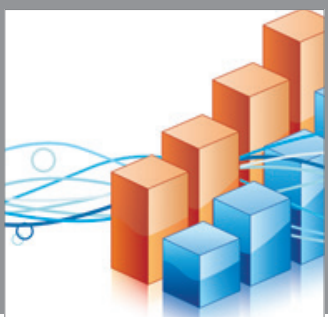

Advances in

Operations Research

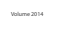

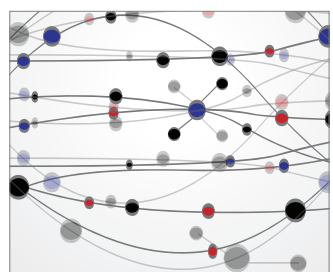

\section{The Scientific} World Journal
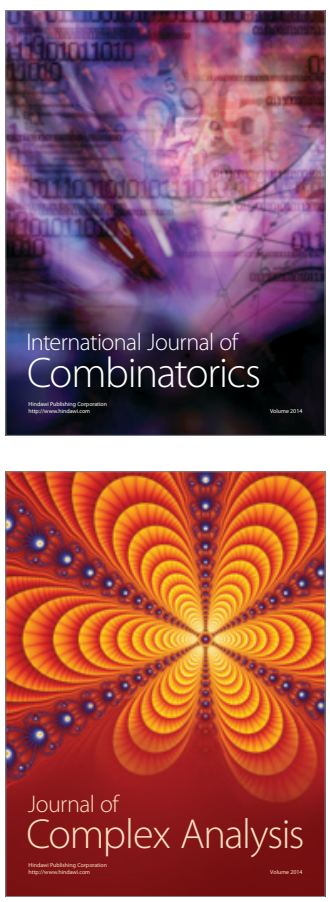

International Journal of

Mathematics and

Mathematical

Sciences
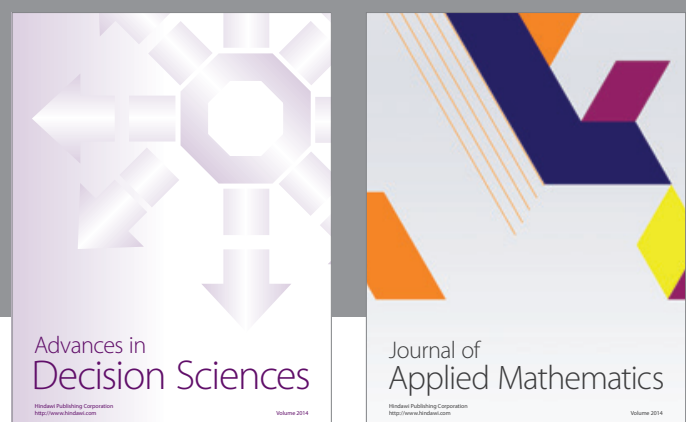

Journal of

Applied Mathematics
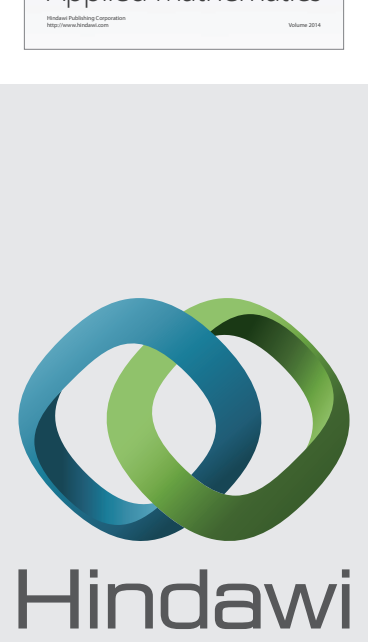

Submit your manuscripts at http://www.hindawi.com
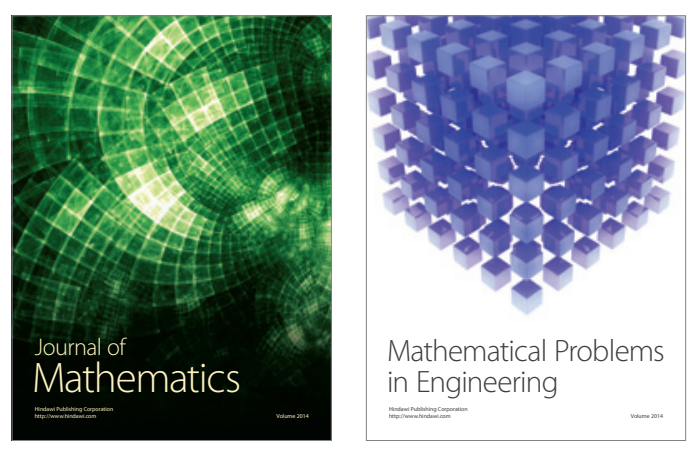

Mathematical Problems in Engineering
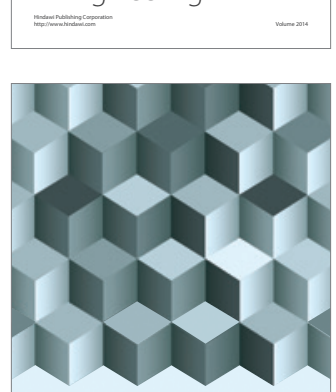

Journal of

Function Spaces
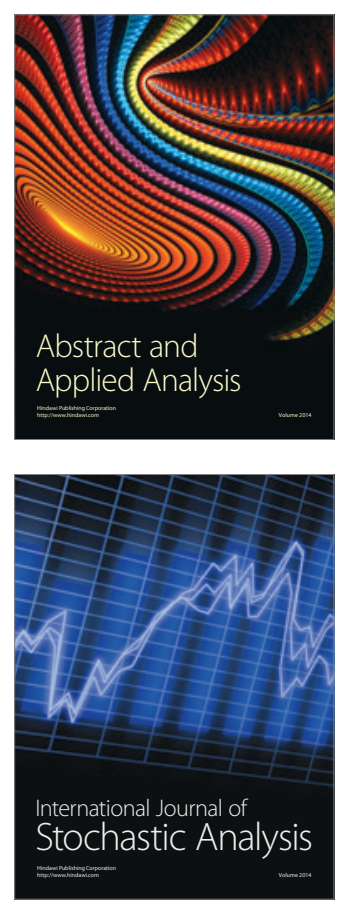

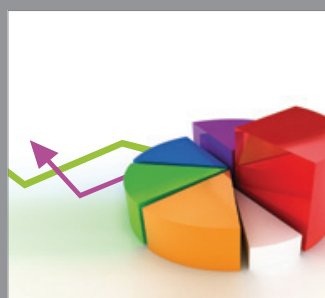

ournal of

Probability and Statistics

Promensencen
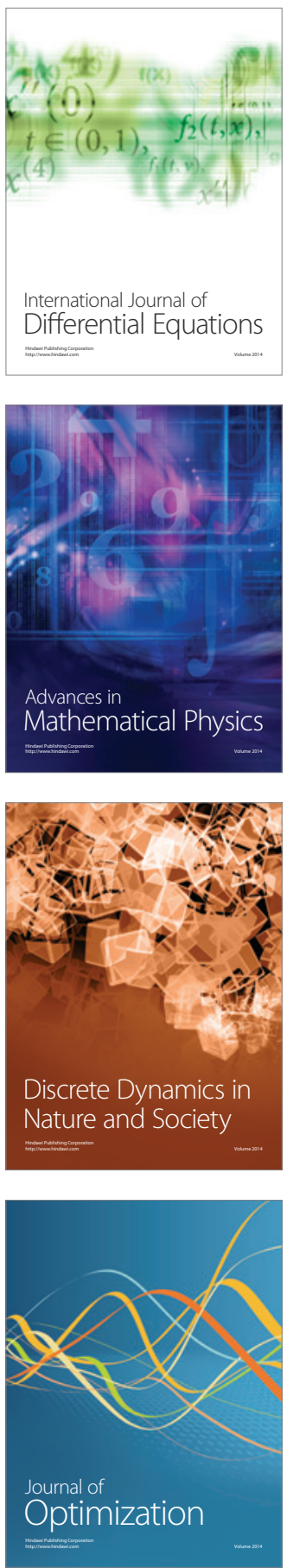